\title{
BMJ open An enhanced exercise and cognitive programme does not appear to reduce incident delirium in hospitalised patients: a randomised controlled trial
}

\author{
Kimberley J Jeffs, ${ }^{1,2}$ David J Berlowitz, ${ }^{3,4,5}$ Shane Grant, ${ }^{6}$ Vicki Lawlor, ${ }^{6}$ \\ Marnie Graco, ${ }^{3}$ Natalie A de Morton, ${ }^{7}$ Judith A Savige, ${ }^{1,2,5}$ Wen K Lim ${ }^{1,2,5}$
}

To cite: Jeffs KJ,

Berlowitz DJ, Grant S, et al. An enhanced exercise and cognitive programme does not appear to reduce incident delirium in hospitalised patients: a randomised controlled trial. BMJ Open 2013;3:e002569. doi:10.1136/bmjopen-2013002569

- Prepublication history for this paper is available online. To view these files please visit the journal online (http://dx.doi.org/10.1136/ bmjopen-2013-002569).

The results of the study were presented at the Australian and New Zealand Society for Geriatric Medicine Annual Scientific Meeting, Melbourne, August 2008.

Received 10 January 2013 Revised 9 May 2013 Accepted 16 May 2013

This final article is available for use under the terms of the Creative Commons Attribution Non-Commercial 2.0 Licence; see http://bmjopen.bmj.com

For numbered affiliations see end of article.

Correspondence to Dr Kimberley Jeffs; kim. jeffs@nh.org.au

\section{ABSTRACT}

Objective: To determine if a programme of progressive resistance exercise, mobilisation and orientation, in addition to usual care, was superior to usual care alone in the prevention of incident delirium in older hospitalised patients.

Design: A randomised controlled trial.

Setting: The study was performed at a secondary referral hospital in Melbourne, Australia between May 2005 and December 2007.

Participants: 648 consecutive medical inpatients aged 65 years or older who had been in hospital for less than $48 \mathrm{~h}$ and who did not have delirium.

Intervention: Participants were randomly allocated to a twice-daily programme of progressive resistance exercise tailored to individual ability, mobilisation and orientation in addition to usual care or to usual care alone.

Measurements: Delirium was measured using the Confusion Assessment Method at baseline and every $48 \mathrm{~h}$ until discharge. Secondary outcome measures were severity and duration of delirium, discharge destination and length of stay.

Results: Delirium occurred in $4.9 \%(95 \% \mathrm{Cl} 2.3 \%$ to $7.3 \%$ ) of the intervention group (15/305) and in 5.9\% (20/339; $95 \% \mathrm{Cl} 3.8 \%$ to $9.2 \%$ ) of the group receiving usual care. No difference was observed between groups $\left(\chi^{2} ; p=0.5\right)$. The intervention had no effect on delirium duration, severity, discharge destination or length of stay.

Conclusion: A programme of progressive resistance exercise and orientation was not effective in reducing incident delirium in hospitalised elderly patients.

\section{INTRODUCTION}

Delirium refers to an acute change in cognition associated with fluctuation, deficits in attention and frequent perceptual changes. It complicates the stay of up to $56 \%$ of older patients admitted to hospital and leads to substantial functional decline, loss of independence,

\section{ARTICLE SUMMARY}

Article focus

- Prevention of delirium is the most promising means of tackling delirium rates, but few rigorous studies have been performed.

- Multicomponent interventions for delirium prevention may be difficult to reproduce.

- This article presents the results of the first randomised controlled trial of delirium prevention in medical inpatients.

Key messages

- A streamlined delirium prevention programme may be readily incorporated into patient care.

- Delirium rates were lower than anticipated, which may relate to a high standard of usual care.

- A programme of twice-daily orientation and mobilisation was not sufficient to reduce incident delirium.

Strengths and limitations of this study

- This randomised controlled trial employed rigorous methods including allocation concealment and blinded assessment of outcomes.

- The low rate of observed delirium meant reduced power to detect any effect of the intervention.

- Co-location of participants in both treatment groups may have led to contamination effects.

psychological distress and persistent cognitive dysfunction. $^{1-4}$ For healthcare systems, this creates the burden of longer hospital stays, increased need for subacute or nursing home care and increased support following discharge..$^{5-8}$

Despite attention to its pathogenesis, risk factors and treatment, delirium prevention remains the most promising means by which to reduce morbidity and healthcare costs. ${ }^{9} 10$ However, no randomised controlled trials of delirium prevention in medical inpatients have been reported. ${ }^{9}{ }^{11}$ A meta-analysis of delirium prevention reported four studies in 
medical inpatients, of which three had contemporaneous controls. ${ }^{11}$ The interventions trialled were educational strategies, reorganisation of care and provision of additional programmes aimed at ameliorating known risk factors for delirium. The results of the meta-analysis suggest that delirium prevention strategies are successful when applied to populations where the delirium rate is over $30 \%$. However, the heterogeneity of both the populations studied (including surgical) and the interventions applied and the poor methodological quality of the studies limits the strength of the results.

In the largest study of delirium prevention, a multicomponent intervention appeared to reduce the rate of incident delirium $(9.9 \%$ vs $15 \%)$ but was not costeffective for those at high risk of delirium. ${ }^{12}{ }^{13}$ Although this intervention was subsequently adopted with modifications in some Australian hospitals, its complex nature and reliance on extensive volunteer networks may have limited its applicability to many hospital situations. ${ }^{14}$ Further, a large controlled study, not included in the meta-analysis, reported that a reorganisation of care in a geriatric care unit could reduce incident delirium $(11.7 \%$ vs $18.5 \%) .{ }^{15}$ However, differences in the baseline characteristics of the participants and of the usual care in the study arms have raised doubts as to the intervention's efficacy. ${ }^{16}$

Secondary analyses of the study by Inouye $e t a l^{17}$ suggested that patient mobilisation and orientation might have been most influential in reducing delirium arising in hospital. These facets of the intervention were applied to all participants and had good adherence and were demonstrated to be associated with a reduction in delirium, whereas other components of the intervention were either not applied to all participants or were poorly adhered to. However, a systematic review suggested that exercise interventions alone were not effective in reducing functional decline, length of stay or need for rehabilitation in hospitalised older patients. ${ }^{18}$ The authors noted that the quality of the evidence was poor and it was unknown whether an intervention that combined physical activity and a simple orienting protocol would be effective. We therefore conducted a large-scale randomised controlled trial of an intervention that aimed to reduce incident delirium by targeting two powerful risk factors: immobilisation and cognitive impairment.

\section{METHODS}

\section{Design overview}

A randomised parallel group controlled trial with a $1: 1$ allocation ratio was performed. The primary outcome measure was episodes of delirium occurring in hospital. Secondary measures were duration of delirium, delirium severity, functional status, discharge destination and length of stay.

Written informed consent was obtained from the patient or substitute decision-maker in the event of cognitive impairment precluding capacity to give consent. Following consent, a baseline assessment was undertaken which included detection of prevalent delirium using the Confusion Assessment Method (CAM). Patients who were found to have delirium at enrolment were not eligible for randomisation and were withdrawn from further participation.

\section{Setting and participants}

The trial was undertaken between May 2005 and December 2007 at a secondary referral hospital in Melbourne. Consecutive patients who met the following criteria were eligible for inclusion: age 65 years or older, admitted to a medical unit in the study area and who had been in hospital for less than $48 \mathrm{~h}$. The following exclusion criteria were applied: severe dysphasia rendering communication impossible, death expected within $24 \mathrm{~h}$, isolation for infection control, documented contraindication to mobilisation, admission to the Stroke Unit or to critical care (intensive or coronary care), planned admission of less than $48 \mathrm{~h}$, major psychiatric diagnosis (eg, schizophrenia), previous inclusion in the study, delirium documented in the admission notes and transfer from another hospital. The Northern Hospital's Human Research and Ethics Committee approved the study (Project 03/05). The study was registered with the Australian Clinical Trials Registry (ACTRN 012605000044628) on 26 July 2005, recruitment having started on 2 May 2005. At that time, investigator-led clinical trials were not yet routinely registered.

\section{Randomisation and interventions}

Participants were randomly assigned either to receive a programme of progressive resistance exercise, mobilisation and orientation in addition to usual care or to receive usual care alone. Allocation concealment was achieved by ensuring that randomisation was performed by a study team member not involved in participant recruitment. Randomisation was achieved using sealed opaque envelopes.

The intervention was delivered twice daily until discharge. Participants were assessed every $48 \mathrm{~h}$ until discharge for delirium, functional status and delirium risk factors. Assessors of outcome measures were blinded to group allocation. Participants and ward staff were not informed of group allocation.

Participants randomised to the intervention arm received a graded physical activity and orientation programme twice daily, which was delivered in addition to usual care. A certified Allied Health Assistant, trained in administering exercise programmes, delivered the intervention after initial assessment of the participant by a physiotherapist. The programme started on the same day as the participant was randomised. Commensurate with ability, participants were prescribed one of four exercise programmes: bed, seated, standing or rails. Gravity, body or light weights were used as resistance as appropriate. Resistance was increased whenever a 
participant could perform 10 repetitions at the previous level. All programmes were customised to the participant's ability and were reviewed daily in order to ascertain if: the programme could be completed safely; the level of difficulty was appropriate to the participant's ability; there had been improvement or deterioration in the participant's condition necessitating a programme change; and if the Allied Health Assistant was having any problems in delivering the programme. Exercise programmes were modified to ensure suitable progression for those participants who made significant gains. Participants received approximately 20-30 min additional therapy per session during weekdays. Suitable participants were encouraged to continue the exercise programme over weekends.

The orientation programme comprised formal and informal elements. The formal element of the programme comprised a series of seven questions aimed at assessing and improving orientation (day, month, year, date, ward, bed number and name of primary nurse). The participant was asked the questions in sequence and prompted with the correct answer if they were not able to give a correct response. The informal element of the programme related to engaging in the exercise programme and in the social interaction with the Allied Health Assistant and/or Physiotherapist. All therapy encounters were recorded and reasons for nonattendance were detailed. Exercise sheets were reviewed daily to monitor adherence to the programme. The amount (in minutes) of therapy received by participants was recorded. All routine physiotherapy and Allied Health Assistant encounters were recorded on a hospital database. This enabled comparison of the therapy received between groups. Neither the physiotherapist nor the Allied Health Assistant had any contact with participants in the usual care group.

Usual care included $24 \mathrm{~h}$ nursing care, daily medical assessment and allied health referral by medical, nursing or other staff. Allied health input was provided on referral only, but daily ward meetings were held to review patient progress and facilitate referrals. Patients with significant functional, cognitive or social issues could be referred to the Aged Care medical consultation service that performed a daily round and could offer advice regarding the recognition, investigation and management of geriatric syndromes including delirium.

\section{Outcomes and follow-up}

The primary outcome measure was the first episode of delirium measured every $48 \mathrm{~h}$ using CAM. ${ }^{19}$ Trained assessors performed CAM in the context of a formal cognitive assessment using the Mini-Mental State Examination and drawing a clock face. ${ }^{20}{ }^{21}$ Delirium severity was measured using a previously described method of scoring delirium symptoms from CAM and duration of delirium was calculated using the first and last assessments where delirium was observed. ${ }^{22}$
Factors predisposing to delirium were assessed at the initial interview. Premorbid cognitive impairment was defined as either a formal documented diagnosis of dementia or a Modified Blessed Dementia Rating scale score $>4 .^{23}{ }^{24}$ Vision was assessed using the Centre for Eye Research Australia kit and hearing assessed using the Whisper test. ${ }^{25}{ }^{26}$ Acute illness severity was measured using the Acute Physiology, Age, and Chronic Health Evaluation II scale and chronic illness burden by the Charlson comorbidity index. ${ }^{27}{ }^{28}$ Functional status was measured using the Modified Barthel Index and the Lawton-Brody Instrumental Activities of Daily Living scale. ${ }^{29} 30$

Power calculations were made using the following assumptions, using data from the large multicomponent trial. ${ }^{12}$ That is, an incident rate of delirium of $15 \%$ with an absolute risk reduction of $6 \%$ (ie, $15 \%$ in the control arm and $9 \%$ in the intervention arm). Assuming a twosided statistical significance of 0.05 and a power of 0.8 , 920 participants would be needed. All analyses were performed on an intention-to-treat basis. Normally distributed data were compared with $\mathrm{t}$ tests and were expressed as mean, SD and 95\% CIs unless otherwise stated. Between-groups comparisons of non-normally distributed data were made with the Mann-Whitney U statistic and within-group comparisons using the Wilcoxon signed rank test. Proportions were compared with Pearson's $\chi^{2}$ test using the continuity correction or with Fisher's exact test where the cell sizes were too small. Statistical significance was set at an $\alpha$ of 0.05 . All missing data were dealt with using the last observation carried forward since the primary outcome measure was binary and not readily amenable to interpolative methods.

The Kaplan-Meier survival analysis was used to determine any effect of the intervention on the time taken to develop incident delirium to test the hypothesis that the intervention delayed the onset of delirium. A Kaplan-Meier analysis was performed using the time between admission and the first assessment with delirium to define the delay to delirium. Those who did not develop delirium were censored at discharge. Cox proportional hazards modelling was used to investigate associations with delay to delirium.

\section{RESULTS}

Over the study period, 3931 admissions meeting the inclusion criteria were screened (figure 1). Of those, 1394 met at least one exclusion criterion and were not eligible for participation. Of the 2537 remaining admissions, 1753 did not participate as the person either declined consent (403) or recruitment could not be completed within $48 \mathrm{~h}$ of hospital admission (1350).

Written consent was given in 784 cases; however, 43 people were unexpectedly discharged or transferred out of the study area prior to a baseline assessment; 54 more withdrew at the baseline assessment. Of the 687 


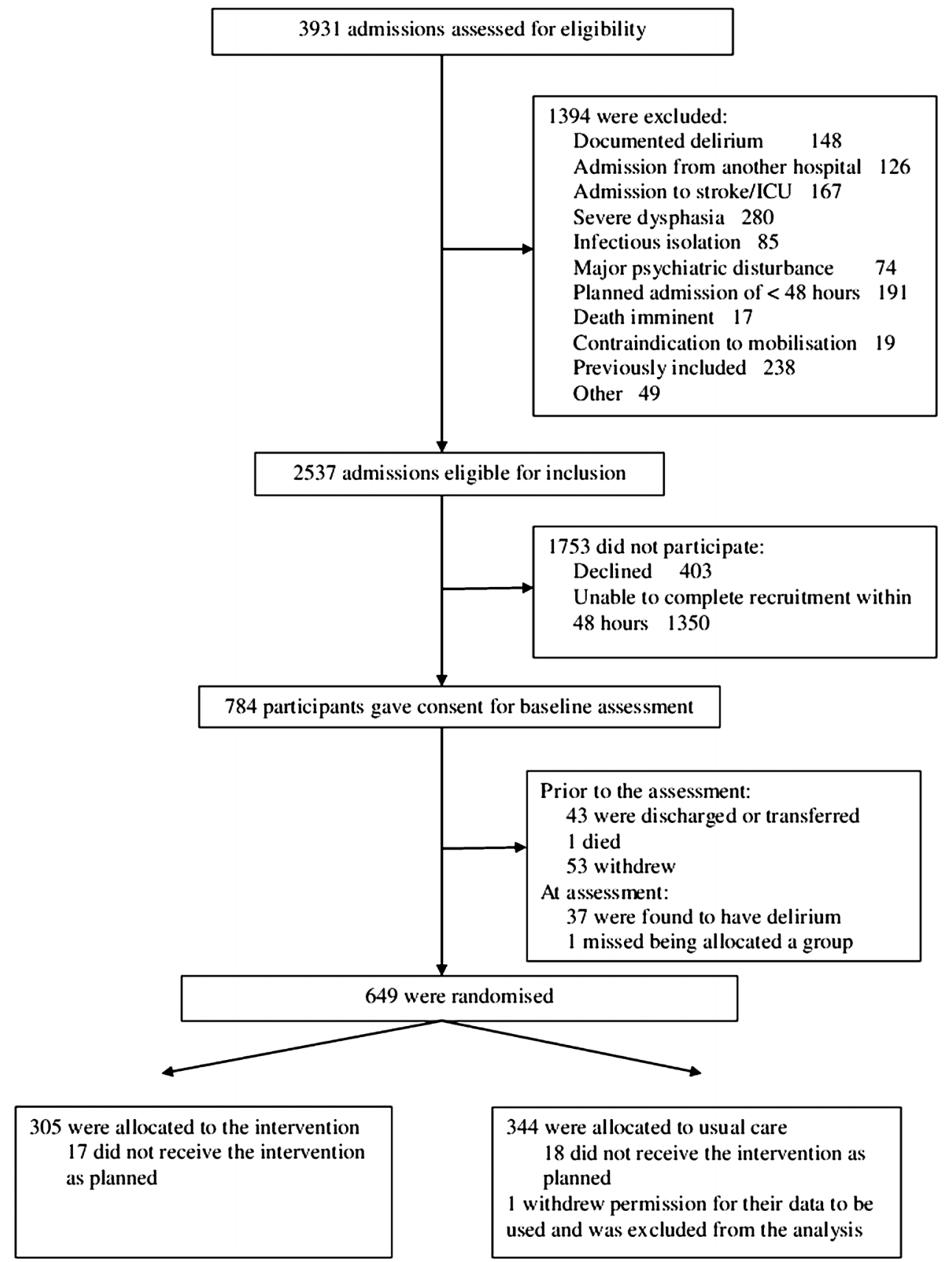

Figure 1 Participant flow diagram.

participants who underwent baseline assessment, 37 were found have delirium. One participant was not allocated to a group due to an administrative error.

At baseline, participants in the two groups were well matched for previously described risk factors for delirium (table 1).

The intervention was delivered as planned. Participants in the intervention arm received a median of $1.4(0.9-1.8)$ therapeutic encounters per day while those in the usual care arm received $0.3(0-0.6)$. Intervention participants received a median of 38 (2552) $\min$ of therapy today compared with $8(0-17)$ for those in the usual care group. This is equivalent to approximately 1.4 additional encounters or $42 \mathrm{~min} /-$ weekday, suggesting good adherence to the protocol, given that therapy was delivered only on weekdays. No adverse events were reported.

Delirium was observed in 36 participants. No effect of the intervention was observed for any of the delirium outcomes, including severity (table 2).

Rates of incident delirium were low and the median duration was short at around two-and-a-half days. Similarly, survival analyses found no difference between the groups $\left(\chi^{2} 0.49 ; p=0.5\right.$ by the $\log$ rank test, see figure 2$)$. At the median length of stay ( 5.5 days), there was no difference in the cumulative incidence of delirium. Cox regression 
Table 1 Baseline characteristics

\begin{tabular}{|c|c|c|}
\hline Characteristic & Intervention $(\mathrm{n}=305)$ & Usual care $(n=343)$ \\
\hline Age (mean, SD) & $79.6(7.5)$ & $79.1(7.9)$ \\
\hline Male $(n, \%)$ & $137(45)$ & $171(50)$ \\
\hline Spoke English at home (n, \%) & $185(61)$ & $224(65)$ \\
\hline Resident in an $\operatorname{RACF}^{\star}(\mathrm{n}, \%)$ & $21(7)$ & $47(14)$ \\
\hline Barthel index† (median, IQR) & $91(71-100)$ & $90(71-100)$ \\
\hline IADL $\ddagger$ (median, IQR) & $6(3-8)$ & $6(3-8)$ \\
\hline Visually impaired§ (n, \%) & $59(20)$ & $83(26)$ \\
\hline Hearing impaired $(n, \%)$ & $61(20)$ & $69(21)$ \\
\hline Premorbid cognitive impairment ${ }^{* *}(n, \%)$ & $38(13)$ & $50(15)$ \\
\hline MMSE score†† (median, IQR) & $25(20-28)$ & $26(19-28)$ \\
\hline 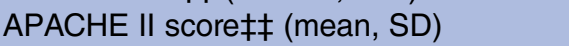 & $14.3(5.0)$ & $14.0(4.6)$ \\
\hline Charlson score§§ (median, IQR) & $2(1-3)$ & $2(1-3)$ \\
\hline Ureaๆๆ (median, IQR) & $8.7(6.5-12.2)$ & $8.0(6.0-12.4)$ \\
\hline Creatinine $^{\star \star \star}$ (median, IQR) & $0.10(0.08-0.13)$ & $0.10(0.08-0.14)$ \\
\hline Urea/creatinine ratio (median, IQR) & $88(70-110)$ & $84(69-104)$ \\
\hline Albumin ${ }^{\star * \star}$ (mean, SD) & $34.5(5.4)$ & $34.4(5.4)$ \\
\hline Oxygen therapy $(n, \%)$ & $68(25)$ & $69(22)$ \\
\hline Intravenous therapy (n, \%) & $36(13)$ & $43(14)$ \\
\hline Indwelling catheter ( $\mathrm{n}, \%)$ & $22(7)$ & $31(9)$ \\
\hline \multicolumn{3}{|c|}{ 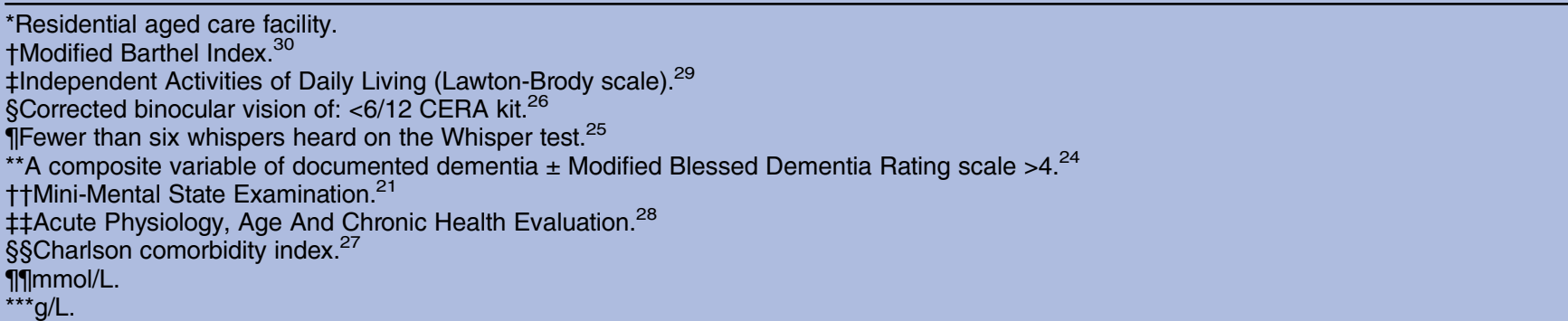 } \\
\hline
\end{tabular}

methods did not reveal any effect of the intervention on duration of delirium (HR 1.2; 95\% CI 0.6 to $2.5 ; \mathrm{p}=0.5$ ) or on length of stay (HR 1.08; 95\% CI 0.9 to 1.3 ; $\mathrm{p}=0.3$ ). Subgroup analysis of those at intermediate to high delirium risk ${ }^{31}$ at admission was performed. All 36 cases of delirium occurred in the 'at risk' group, equating to a rate of $6.8 \%$ (95\% CI $4.8 \%$ to $9.8 \%$ ). Rates of delirium were not different between the groups (intervention, 15/249 vs control 21/280; $p=0.6)$. The length of stay and discharge outcomes were not significantly influenced by group allocation.

\section{DISCUSSION}

An intervention incorporating a progressive resistance exercise programme, mobilisation and orientation was not effective in reducing delirium arising in hospital. Nor did the intervention improve the functional, cognitive or discharge outcomes for patients. Additional physical activity and orientation did not reduce the rate, duration or severity of incident delirium or delay the onset of delirium. No effect of the programme was observed for functional or cognitive outcomes and there was no evidence of functional decline in either group.

\section{Table 2 Outcomes}

\begin{tabular}{lccc}
\hline Characteristic & Intervention $(\mathbf{n = 3 0 5 )}$ & Usual care $(\mathbf{n = 3 4 3 )}$ & $\mathbf{p}$ Value \\
\hline Episodes of delirium (n, \%) & $15(4.9)$ & $21(5.9)$ & See text \\
Duration of delirium (days, median, IQR) & $2.4(0.9-8.9)$ & $2.1(1.4-6.6)$ & $0.9^{*}$ \\
Severity of delirium (median, IQR) & $3.0(3.0-5.0)$ & $4.0(3.0-4.5)$ & $0.7^{*}$ \\
Length of stay (days, median, IQR) $\dagger$ & $5.5(3.9-9.2)$ & $5.6(3.3-9.0)$ & See text \\
Return to previous residence (n, \%) & $221(75)$ & $258(79)$ & $0.4 \S$ \\
Lowest, baseline BIף (median, IQR) & $0(-1-2)$ & $0(-1-0)$ & $0.3^{*}$ \\
\hline
\end{tabular}

*Mann-Whitney $U$ test.

†Those who died excluded: $\mathrm{n}=293$ and 328 , respectively, data missing for 11 participants.

$\S$ Pearson's $\chi^{2}$.

$\mathrm{qB} \mid=$ modified Barthel Index. ${ }^{30}$ 


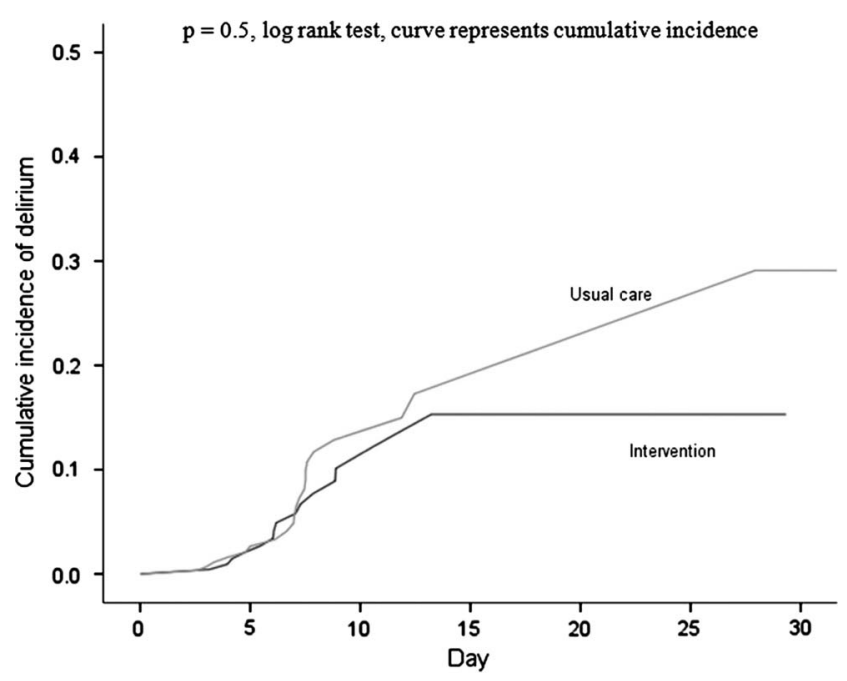

Figure 2 Kaplan-Meier analysis of delay to delirium.

The intervention did not reduce the length of stay or alter the discharge outcomes.

The strengths of this study include its large size and rigorous methodology including prospective individual randomisation, allocation concealment, blinded assessment of outcomes, complete follow-up and intention-to-treat analysis. Furthermore, we were able to demonstrate that the intervention was delivered as planned.

Low rates of incident delirium were observed in both arms of the trial compared with the previous literature reporting delirium prevention. ${ }^{9} 11121415$ Possible explanations include that 48 hourly assessment for delirium missed cases or that, unlike earlier studies, our participants were not selected for delirium risk. An alternative hypothesis is that rates of delirium are truly lower in this setting than in others described previously. The median duration of delirium was more than 2 days and the research nurse had access to corroborative history from staff caring for the participant and family members in order to score the items of acute change and fluctuation. Thus, it appears unlikely that the reduced frequency of measurement significantly underestimated delirium incidence. All patients, regardless of the baseline delirium risk, were eligible for inclusion in the study, as previous work had suggested that $80 \%$ of patients at the study hospital were at intermediate to high risk of delirium. ${ }^{6}$ Of those enrolled, $468(72 \%)$ were at intermediate risk and $61(9 \%)$ at high risk, consistent with the previous estimate. Despite studying an unselected population, the prevalence of delirium risk factors in our participants closely matched those of the largest multicomponent trial (table 3). Incident delirium rates in a subgroup aged $>70$ and at intermediate to high risk were also lower $(7.8 \%)$ than the rates reported previously $(12.4 \%)$. The rates of delirium observed in both arms of this study were clinically significantly lower than those in the usual care arm of the Inouye trial and not different from the intervention arm in that study (table 4). Thus, it seems possible that incident delirium rates are lower in our Australian hospital than those described elsewhere.

Previous literature has not identified which features of a multicomponent intervention are most associated with its effect. Our results may suggest that the wrong components of the intervention were tested, all elements of a multicomponent intervention were required or that the positive results observed in previous studies were due to study design or chance. Even in the largest and most methodologically rigorous study, the positive effect observed may have been due to uncontrolled contamination or observation biases secondary to the prospectively matched design. Previous research suggests that the greatest benefit of delirium prevention strategies is for those at intermediate risk of delirium (OR for delirium by treatment group $0.52,95 \%$ CI 0.29 to 0.92$).{ }^{12}$ A welldesigned, prospective randomised controlled trial recruiting primarily those at intermediate risk would therefore be expected to find an effect if there were any true benefits to be derived. The effect size for those at

Table 3 Comparison of baseline characteristics between this and a related study

\begin{tabular}{|c|c|c|c|c|}
\hline \multirow[b]{2}{*}{ Characteristic } & \multicolumn{2}{|l|}{ Intervention } & \multicolumn{2}{|l|}{ Usual care } \\
\hline & Jeffs et al $(n=305)$ & Inouye et al $(n=426)$ & Jeffs et al $(n=343)$ & Inouye et al $(n=426)$ \\
\hline Age (mean, SD) & $79.6(7.5)$ & $79.6(6.1)$ & $79.1(7.9)$ & $79.8(6.2)$ \\
\hline Male $(n, \%)$ & $137(45)$ & $167(39)$ & $171(50)$ & $167(39)$ \\
\hline Resident in an $\mathrm{RACF}^{\star}(\mathrm{n}, \%)$ & $21(7)$ & $24(6)$ & 47 (14) & $27(6)$ \\
\hline APACHE II score $†$ (mean, SD) & $14.3(5.0)$ & $15.5(4.0)$ & $14.0(4.6)$ & $15.6(4.1)$ \\
\hline MMSE scoreł & 25 (20 to 28$)$ & $24(4.6)$ & 26 (19 to 28$)$ & $23(4.9)$ \\
\hline Cognitive impairment§ (n, \%) & $38(13)$ & $50(12)$ & $50(15)$ & $45(11)$ \\
\hline Visually impaired $\emptyset$ (n, \%) & $59(20)$ & 97 (23) & $83(26)$ & $98(23)$ \\
\hline Hearing impaired ${ }^{* \star}(n, \%)$ & $61(20)$ & $120(28)$ & $69(21)$ & $98(23)$ \\
\hline
\end{tabular}

${ }^{*}$ Resident in a Residential Aged Care Facility for Jeffs et al; resident in a nursing home for Inouye et al.

†Acute Physiology, Age And Chronic Health Evaluation II. ${ }^{28}$

$\ddagger$ Median and IQR for Jeffs et al; Mean (SD) for Inouye et al, Mini-Mental State Examination (MMSE). ${ }^{21}$

$\S$ Prior cognitive impairment. A composite variable for Jeffs et al of documented dementia \pm Modified Blessed Dementia Rating scale $>4$, for Inouye et al Modified Blessed Dementia Rating scale>2. ${ }^{24}$

ๆCorrected binocular vision of: $<6 / 12$ CERA kit for Jeffs et al; $<20 / 70$ standard Snellen chart. ${ }^{26}$

${ }^{* *}$ Fewer than six whispers heard on the Whisper test. ${ }^{25}$ 
Table 4 Comparison of rates of delirium between the current study and that by Inouye et al *

\begin{tabular}{|c|c|c|c|c|}
\hline Current study & & Inouye et al & & p Value \\
\hline Usual care & & Usual care & & 0.02 \\
\hline Delirium rate & $18 / 235$ & Delirium rate & $62 / 426$ & \\
\hline$\%(95 \% \mathrm{Cl})$ & 7.7 (4.3 to 11.1$)$ & $\%(95 \% \mathrm{Cl})$ & 15.0 (11.6 to 18.4$)$ & \\
\hline Usual care & & Intervention & & 0.5 \\
\hline Delirium rate & $18 / 235$ & Delirium rate & $42 / 426$ & \\
\hline$\%(95 \% \mathrm{Cl})$ & 7.7 (4.3 to 11.1$)$ & $\%(95 \% \mathrm{Cl})$ & 9.9 (7.0 to 12.7$)$ & \\
\hline Intervention & & Intervention & & 0.09 \\
\hline Delirium rate & $12 / 226$ & Delirium rate & $42 / 426$ & \\
\hline$\%(95 \% \mathrm{Cl})$ & 5.3 (2.4 to 8.2 ) & $\%(95 \% \mathrm{Cl})$ & 9.9 (7.0 to 12.7$)$ & \\
\hline
\end{tabular}

intermediate risk in the current study was substantially less than that observed in the Inouye study (OR for delirium by treatment group $0.72,95 \%$ CI 0.33 to 1.59 ).

Intervention and control patients were housed in the same wards, which may have led to contamination effects due to increased referrals for physiotherapy in the control arm. However, we report documented evidence that participants in the intervention arm received an additional 40 min of therapy every weekday.

Although the intervention employed in the current study included an orientation protocol and participation in the physical component was likely to have involved further cognitive stimulation, this may have been insufficient to address cognitive impairment. However, there is only limited evidence that cognitive impairment is a modifiable risk factor. ${ }^{12} 17$

Although statistical power was reduced by the low rate of delirium in this study, it is likely that the intervention was not effective. Unfortunately, we were unable to achieve our a priori target sample size in the time and with the resources available. However, we speculate that given our markedly lower observed rates of incident delirium in both study arms, even if we had randomised 920 participants as planned, we would still have failed to observe any difference. The study was powered for the primary outcome measure of the first episode of incident delirium and power calculations were based on estimates of delirium rates of $15 \%$ and $10 \%$, respectively. The effect size suggested by the results of this study was less than that found by Inouye (35\% absolute risk reduction). The sample size required to demonstrate with statistical confidence the effect size observed in the current study with the same delirium rates would be close to 16500 participants. In addition, if the observed effect size had to be confirmed in a larger study, the results would indicate that 100 people would need to be treated for every case of delirium prevented.

Controversy exists in the literature regarding the optimal methods for dealing with missing values in longitudinal data sets. We employed a conservative method of data imputation based on clinical and statistical considerations. Missing endpoint data occurred when participants were discharged rapidly from hospital. These participants had a lower baseline risk for delirium and hence were assumed to have remained free of delirium in their brief hospital stay. Analysis with cases of missing censored data did not alter the results.

Our results cast doubt on the efficacy of nonpharmacological interventions in the prevention of hospital-acquired delirium. Future research should focus on rigorous evaluation of multicomponent interventions as applied in real-world settings, the value of screening for delirium risk prior to intervention and on the best methods for evaluating health service research in vulnerable populations.

\section{Author affiliations}

${ }^{1}$ Northern Health, Melbourne, Victoria, Australia

${ }^{2}$ Department of Medicine, Dentistry and Health Sciences, The University of Melbourne, Melbourne, Victoria, Australia

${ }^{3}$ Department of Respiratory and Sleep Medicine, Institute for Breathing and Sleep, Austin Health, Melbourne, Victoria, Australia

${ }^{4}$ Department of Respiratory and Sleep Medicine, Austin Health, Melbourne, Victoria, Australia

${ }^{5}$ Department of Medicine, Austin Health and Northern Health, Melbourne, Victoria, Australia

${ }^{6}$ The Northern Clinical Research Centre, Northern Health, Melbourne, Victoria, Australia

${ }^{7}$ Donvale Rehabilitation Hospital, Ramsay Health, Melbourne, Victoria, Australia

Acknowledgements The authors would like to thank the staff and patients of The Northern Hospital for their assistance with this study. The study team comprised Shane Grant, Vicki Lawlor, Dorothy Lewis, Marnie Graco, Natalie de Morton and Laura Wickett.

Contributors KJJ was responsible for the study design, data collection, analysis and interpretation and manuscript preparation. DJB provided advice regarding the study design, data analysis and manuscript preparation. WKL advised regarding the study design, data analysis and assisted with manuscript preparation. SG, VL, $M G$ and NADM were involved in refinements to the study protocol, data collection, data preparation and manuscript review. JAS was involved in manuscript preparation. All authors have read and approved the final manuscript.

Funding The Northern Clinical Research Centre, Northern Health and The University of Melbourne provided institutional support. The study was supported by a grant from the HCF Health and Medical Research Foundation (Effect of progressive exercise training on mobility, cognition and healthcare utilisation for acutely hospitalised older patients). The Department of Human Services VIC provided institutional funding support. KJJ and NADM were supported by the National Health and Medical Research Council (KJJ: 
Scholarship 310631, NADM: Scholarship 280632, Fellowship 551955). The sponsors had no role in the study design, participant recruitment, data collection, data analysis or manuscript preparation.

Competing interests None.

Ethics approval The Northern Hospital's Human Research and Ethics Committee approved the study (Project 03/05).

Provenance and peer review Not commissioned; externally peer reviewed.

Data sharing statement Original dataset and supplementary analyses may be provided by consultation with the principal author.

\section{REFERENCES}

1. O'Keeffe S, Lavan J. The prognostic significance of delirium in older hospital patients. J Am Geriatr Soc 1997;45:174-8.

2. Inouye SK. Delirium in hospitalized older patients: recognition and risk factors. J Geriatr Psychiatry Neurol 1998;11:118-25; discussion 57-8.

3. Duppils GS, Wikblad K. Patients' experiences of being delirious. J Clin Nurs 2007;16:810-18.

4. O'Malley G, Leonard M, Meagher D, et al. The delirium experience: a review. J Psychosom Res 2008;65:223-8.

5. Adamis D, Treloar A, Martin FC, et al. Recovery and outcome of delirium in elderly medical inpatients. Arch Gerontol Geriatr 2006;43:289-98.

6. Jeffs K, Lim W, Berlowitz D, et al. Delirium in a culturally diverse medical inpatient population 2008; Fremantle. Intern Med J 2008;38 (Suppl 5):A121.

7. McCusker J, Cole M, Dendukuri N, et al. Delirium in older medical inpatients and subsequent cognitive and functional status: a prospective study. CMAJ 2001;165:575-83.

8. McCusker J, Cole MG, Dendukuri N, et al. Does delirium increase hospital stay? J Am Geriatr Soc 2003;51:1539-46.

9. Tabet N, Howard R. Non-pharmacological interventions in the prevention of delirium. Age Ageing 2009;38:374-9.

10. Cole MG, McCusker J, Bellavance F, et al. Systematic detection and multidisciplinary care of delirium in older medical inpatients: a randomized trial. CMAJ 2002:167:753-9.

11. Hempenius L, Van Leeuwen BL, Van Asselt DZ, et al. Structured analyses of interventions to prevent delirium. Int J Geriatr Psychiatry 2011;26:441-50

12. Inouye SK, Bogardus ST Jr, Charpentier PA, et al. A multicomponent intervention to prevent delirium in hospitalized older patients. N Engl J Med 1999;340:669-76.

13. Rizzo JA, Bogardus ST Jr, Leo-Summers L, et al. Multicomponent targeted intervention to prevent delirium in hospitalized older patients: what is the economic value? Med Care 2001;39:740-52.

14. Caplan GA, Harper EL. Recruitment of volunteers to improve vitality in the elderly: the REVIVE study. Intern Med J 2007;37:95-100.

15. Vidan MT, Sanchez E, Alonso M, et al. An intervention integrated into daily clinical practice reduces the incidence of delirium during hospitalization in elderly patients. J Am Geriatr Soc 2009;57:2029-36.

16. Inouye SK, Rubin FH, Wierman HR, et al. No shortcuts for delirium prevention. J Am Geriatr Soc. [Comment Letter] 2010;58:998-9; author reply 9-1000.

17. Inouye SK, Bogardus ST Jr, Williams CS, et al. The role of adherence on the effectiveness of nonpharmacologic interventions: evidence from the delirium prevention trial. Arch Intern Med 2003;163:958-64.

18. De Morton NA, Keating JL, Jeffs K. The effect of exercise on outcomes for older acute medical inpatients compared with control or alternative treatments: a systematic review of randomized controlled trials. Clin Rehabil 2007;21:3-16.

19. Inouye SK, Van Dyck $\mathrm{CH}$, Alessi CA, et al. Clarifying confusion: the confusion assessment method. A new method for detection of delirium. Ann Intern Med 1990;113:941-8.

20. Fisher BW, Flowerdew G. A simple model for predicting postoperative delirium in older patients undergoing elective orthopedic surgery. J Am Geriatr Soc 1995;43:175-8.

21. Folstein MF, Folstein SE, McHugh PR. "Mini-mental state". A practical method for grading the cognitive state of patients for the clinician. J Psychiatr Res 1975;12:189-98.

22. Wei LA, Fearing MA, Sternberg EJ, et al. The confusion assessment method: a systematic review of current usage. J Am Geriatr Soc 2008;56:823-30.

23. Inouye SK, Viscoli CM, Horwitz RI, et al. A predictive model for delirium in hospitalized elderly medical patients based on admission characteristics. Ann Intern Med 1993;119:474-81.

24. Kay DWK. The epidemiology and identification of brain deficit in the elderly. In: Eisdorfer C, Friedel RO (Eds). Cognitive and emotional disturbance in the elderly. Chicago: Year Book Medical Publishers Inc, 1977, pp 11-26.

25. Macphee GJ, Crowther JA, McAlpine $\mathrm{CH}$. A simple screening test for hearing impairment in elderly patients. Age Ageing 1988;17:347-51.

26. Nottle HR, McCarty CA, Hassell JB, et al. Detection of vision impairment in people admitted to aged care assessment centres. Clin Experiment Ophthalmol 2000;28:162-4.

27. Charlson ME, Pompei $\mathrm{P}$, Ales $\mathrm{KL}$, et al. A new method of classifying prognostic comorbidity in longitudinal studies: development and validation. J Chronic Dis 1987;40:373-83.

28. Knaus WA, Draper EA, Wagner DP, et al. APACHE II: a severity of disease classification system. Crit Care Med 1985; 13:818-29.

29. Lawton MP, Brody EM. Assessment of older people: self-maintaining and instrumental activities of daily living. Gerontologist 1969:9:179-86.

30. Shah S, Vanclay F, Cooper B. Improving the sensitivity of the Barthel Index for stroke rehabilitation. J Clin Epidemiol 1989;42:703-9.

31. Inouye SK. Predisposing and precipitating factors for delirium in hospitalized older patients. Dement Geriatr Cogn Disord 1999;10:393-400. 